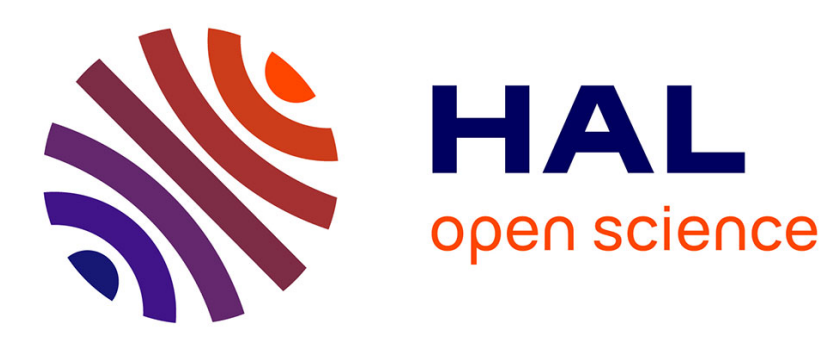

\title{
Ségrégation intergranulaire des éléments de la famille du soufre dans le fer pur
}

\author{
Capucine Pichard, Michel Guttmann, J. Rieu, C. Goux
}

\section{To cite this version:}

Capucine Pichard, Michel Guttmann, J. Rieu, C. Goux. Ségrégation intergranulaire des éléments de la famille du soufre dans le fer pur. Journal de Physique Colloques, 1975, 36 (C4), pp.C4 151-C4 155. 10.1051/jphyscol:1975416 . jpa-00216321

\section{HAL Id: jpa-00216321 https://hal.science/jpa-00216321}

Submitted on 1 Jan 1975

HAL is a multi-disciplinary open access archive for the deposit and dissemination of scientific research documents, whether they are published or not. The documents may come from teaching and research institutions in France or abroad, or from public or private research centers.
L'archive ouverte pluridisciplinaire HAL, est destinée au dépôt et à la diffusion de documents scientifiques de niveau recherche, publiés ou non, émanant des établissements d'enseignement et de recherche français ou étrangers, des laboratoires publics ou privés. 


\title{
SÉGRÉGATION INTERGRANULAIRE DES ÉLÉMENTS DE LA FAMILLE DU SOUfRe DANS LE FER PUR
}

\author{
C. PICHARD $(*)$, M. GUTTMANN $(* *)$, J. RIEU $\left({ }^{*}\right)$ et C. GOUX $(*)$
}

\begin{abstract}
Résumé. - Les mécanismes de ségrégation intergranulaire des éléments soufre, sélénium et tellure ont été étudiés en utilisant d'une part nos propres mesures de ségrégation obtenues par rétrodiffusion élastique d'ions accélérés dans le cas des éléments sélénium et tellure, d'autre part quelques résultats de la littérature obtenus par spectroscopie Auger dans le cas des ségrégations de soufre.

L'ensemble des résultats montre que la ségrégation intergranulaire des trois métalloïdes obéit aux mêmes lois. Il s'agit essentiellement d'une ségrégation d'équilibre, réversible et activée thermiquement qui n'est pas modifiée par la présence de carbone ségrégé aux joints de grains.

Une équation générale est proposée. Elle permet de décrire nos résultats ainsi que certains résultats de la littérature.
\end{abstract}

Abstract. - The factors involved in the intergranular segregation of solute elements such as sulphur, selenium and tellurium have been investigated using, on the one hand, elastic backscattering of energetic ions in the case of selenium and tellurium, and the basis of published data obtained by Auger electron spectroscopy in the case of sulphur on the other hand.

The results so obtained show that intergranular segregation of the three metalloidic elements follows the same laws. The process is basically an equilibrium segregation, reversible and thermally activated. which is not modified by carbon segregation at the grain boundaries.

A general equation is proposed which can account for our own results as wall as for those found in the litterature.

Il est maintenant bien établi que la plupart des cas de fragilité intergranulaire du fer et de ses alliages est due à la présence d'impuretés ségrégées aux joints de grains $[1,2,3,4]$. Ainsi dans une précédente étude $[5,6]$ nous avions montré que la présence, en faible quantité, des métallö̈des soufre, sélénium et tellure provoque une fragilité intergranulaire très importante dans un fer de haute pureté préparé au laboratoire. Il apparaissait de plus que cette fragilité intergranulaire était en rapport direct avec la quantité de ces métalloïdes ségrégés aux joints de grains, que la présence ou non d'oxygène ne jouait aucun rôle, mais que par contre le carbone avait un rôle bénéfique sur la cohésion des joints de grains.

La compréhension des phénomènes de fragilité du fer et des paramètres qui l'influencent nécessite donc la connaissance des mécanismes de ségrégation intergranulaire. Cette étude est ainsi consacrée aux résultats de mesures de ségrégations intergranulaires des éléments de la famille du soufre dans le fer pur. La plupart de nos mesures de ségrégations

(*) Département de Métallurgie de l'Ecole Nationale Supérieure des Mines de Saint-Etienne, 158 bis, Cours Faureil, 42023 Saint-Etienne Cedex, France.

$\left({ }^{*}\right)$ Centre des matériaux de l'Ecole Nationale Supérieure des Mines de Paris, BP 87, 91003 Evry Cedex, France. intergranulaires ont été effectuées par la méthode de rétrodiffusion élastique d'ions accélérés que nous n'exposerons pas ici mais qui est abondamment décrite dans la littérature $[7,8]$. Cette méthode présentant une sensibilité d'autant meilleure que le soluté a une masse atomique plus grande que la matrice, nos propres résultats ont été limités aux ségrégations intergranulaires de sélénium et de tellure.

1. Influence de la teneur globale en sélénium et tellure après trempe à partir d'une température donnée. - La fragilité intergranulaire du fer pur augmentant avec sa teneur globale en sélénium et tellure $[5,6]$, nous avons tout d'abord mesuré les ségrégations intergranulaires de ces éléments dans des fers ayant subi un maintien de 3 heures à $800{ }^{\circ} \mathrm{C}$ arrêté par trempe à l'eau. La figure 1 montre les résultats obtenus. Les ségrégations intergranulaires y sont exprimées conventionnellement en pourcentage atomique ramené à une monocouche de fer. On constate que les ségrégations de sélénium et de tellure augmentent avec la teneur globale du fer en ces éléments. Cette croissance se fait suivant une courbe en $\mathrm{S}$ analogue aux courbes taux de recouvrement/pression obtenues dans le cas de l'absorption d'un gaz à la surface d'un métal [9]. En outre la figure 1 montre 


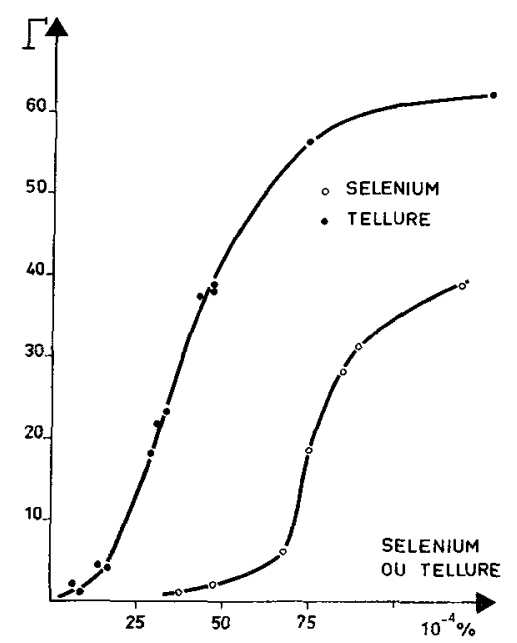

Fıg. 1. - Ségrégation intergranulaire du sélénium et du tellure en fonction de leur teneur globale dans le fer pur trempé depuis $800^{\circ} \mathrm{C}$.

que, pour les plus fortes teneurs en sélénium ou tellure, les ségrégations tendent vers une limite au moins égale à $50 \%$ de monocouche. Cette limite correspond vraisemblablement à la saturation des joints de grains en impuretés fragilisantes lorsque celles-ci atteignent leur limite de solubilité dans le fer pur et commencent à précipiter dans les joints. Rappelons qu'une limite analogue avait été obtenue pour la température de transition du fer pur fragilisé par de petites additions de soufre [6]. Ce résultat souligne à nouveau l'étroite relation qui existe entre la fragilité intergranulaire du fer et les ségrégations intergranulaires qui la provoque.

2. Influence de la température de trempe à teneur en sélénium ou tellure donnée. - On sait que la fragilité intergranulaire d'un fer de composition donnée dépend de la température à laquelle il a été traité $[10,11]$. On doit donc s'attendre ici à d'importantes variations de ségrégation intergranulaire avec la température de trempe du fer.

Pour vérifier ce point nous avons mesuré les ségrégations intergranulaires obtenues pour diverses températures de trempe dans des fers purs à teneur donnée en sélénium ou tellure. Pour chaque température les temps ont été calculés de telle sorte qu'un atome de sélénium ou de tellure situé au centre d'un grain puisse atteindre les joints par diffusion au cours du traitement.

Sur la figure 2 ont été portés les logarithmes des ségrégations de tellure, exprimées en pourcentage de monocouche $\Gamma$, en fonction de l'inverse des températures absolues de maintien. Il apparaît que :

- à une température donnée, la ségrégation augmente avec la teneur globale du fer en tellure;

- pour une teneur donnée en tellure, la ségrégation crô̂t lorsque la température de maintien diminue. Cette croissance étant linéaire dans le système de coordonnées $(\log \Gamma ; 1 / T)$, il en résulte

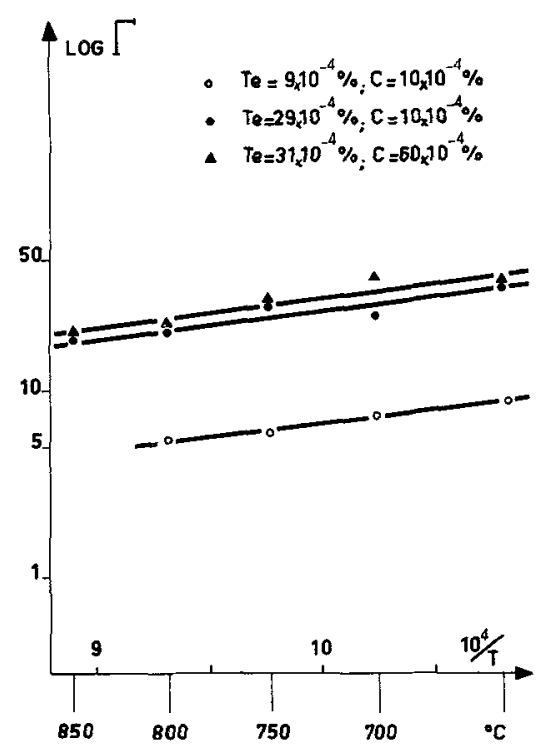

FIG. 2. - Influence de la température de trempe sur la ségrégation intergranulaire du tellure dans le fer pur.

que les ségrégations de tellure suivent une loi d'activation thermique. Le calcul montre que l'énergie d'activation corréspondante est voisine de $15 \mathrm{kcal} / \mathrm{mole}$.

On doit noter ici que, comme on le verra par la suite, les ségrégations de tellure sont les mêmes dans le cas de fers ayant pratiquement même teneur en tellure et des teneurs en carbone très différentes.

3. Etat d'équilibre des ségrégations. - Au vu de ces résultats, il importait de savoir si les niveaux de ségrégation obtenus correspondaient ou non à un équilibre. On sait en effet que certaines ségrégations intergranulaires sont thermodynamiquement hors d'équilibre [12]. Or, une ségrégation est dite d'équilibre si, à température donnée, elle atteint un niveau constant à partir d'un temps donné [13]. Nous avons donc étudié la variation des ségrégations, à teneur en sélénium ou tellure constante, en fonction $\mathrm{du}$ temps de maintien à diverses températures.

La figure 3 est ainsi relative à un fer pur contenant $28 \times 10^{-4} \%$ de tellure trempé à l'eau, après divers maintiens à $800^{\circ} \mathrm{C}$. On constate que, aux erreurs expérimentales près qui sont ici d'environ $1,5 \%$ de monocouche, le niveau des ségrégations intergranulaires de tellure ne varie pas pour des temps de maintien supérieurs à 3 heures. Un équilibre stable est alors atteint.

Les résultats sont analogues pour les ségrégations intergranulaires de sélénium ou pour d'autres températures de maintien. Il apparaît donc que la ségrégation des éléments sélénium et tellure est un phénomène d'équilibre.

4. Influence des conditions de refroidissement. Réversibilité de l'état d'équilibre. - Jusqu'ici tous les résultats ont été obtenus sur des fers purs ayant 


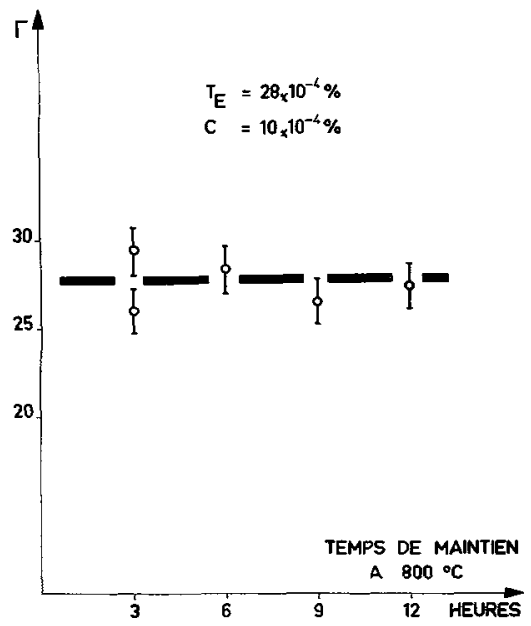

FIG 3. - Influence du temps de maintien sur la ségrégation intergranulaire du tellure dans le fer pur trempé depuis $800^{\circ} \mathrm{C}$.

été trempés à l'eau après traitement thermique. Cependant plusieurs auteurs ont montré l'influence importante des conditions de refroidissement sur la fragilité intergranulaire du fer $[14,15]$. On doit donc s'attendre, pour une température de maintien donnée, à un changement du niveau des ségrégations intergranulaires suivant les conditions de refroidissement.

Les figures 4 et 5 comparent ainsi les ségrégations de sélénium et de tellure obtenues pour des fers ayant subi un maintien de 3 heures à $800^{\circ} \mathrm{C}$ suivi soit d'une trempe à l'eau, soit d'un refroidissement lent au four en 24 heures. On y remarque que, dans tous les cas, les ségrégations intergranulaires sont plus importantes après refroidissement lent qu'après trempe, mais que la limite supérieure est la même.

$\mathrm{Ce}$ dernier point confirme que, comme nous l'avions supposé, c'est la saturation des joints de grains et non les conditions de traitement thermique qui impose cette limite.

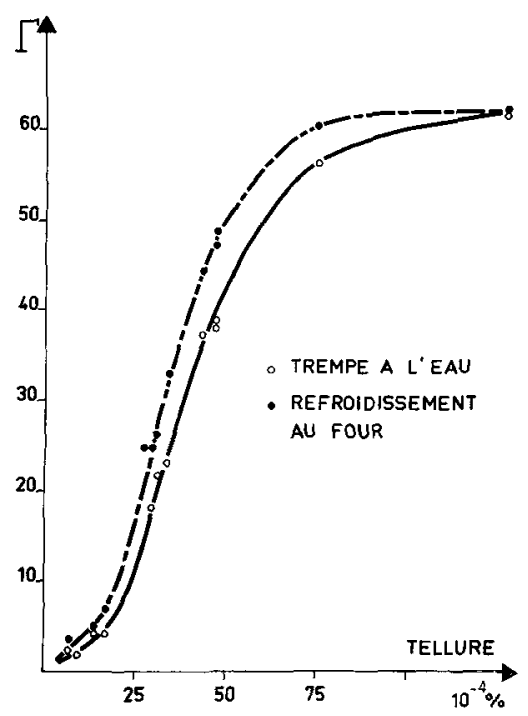

FIG. 4. - Influence des conditions de refroidissement à partir de $800{ }^{\circ} \mathrm{C}$ sur la ségrégation intergranulaire du tellure.

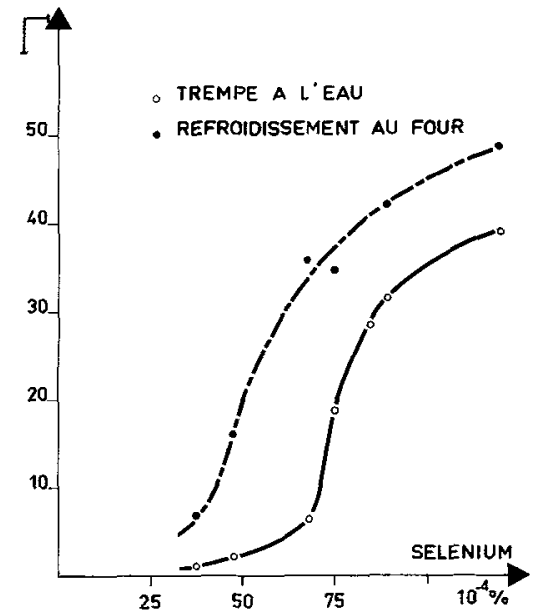

FIG 5. - Influence des conditions de refroidissement à partir de $800^{\circ} \mathrm{C}$ sur la ségrégation intergranulaire du sélénium.

On pourrait penser ici que ces résultats sont en contradiction avec l'existence d'un équilibre, car on connaît l'importance que joue la vitesse de refroidissement dans les cas de ségrégations hors d'équilibre [12]. En fait il n'en est rien. Nous avons vu en effet que les ségrégàtions intergranulaires de sélénium et de tellure augmentent lorsque la température de maintien diminue. Ainsi, au cours d'un refroidissement lent au four, les joints continuent à s'enrichir en impuretés au fur et à mesure que la température décroît, ce qui conduit à un niveau de ségrégations plus important qu'après trempe.

Pour être plus précis nous avons été amenés à étudier les conditions de réversibilité de l'état d'équilibre. Nous avons pour cela comparé les ségrégations intergranulaires obtenues dans un même fer, traité à $800^{\circ} \mathrm{C}$, et ayant subi soit une trempe directe, soit un refroidissement au four suivi à nouveau d'un traitement de trempe. Le tableau I montre les résultats obtenus.

\section{TABLEAU I}

$$
\begin{aligned}
& \text { Impuretés Traitements (\% de } \\
& (10-\%) \text { thermiques monocouche) } \\
& \text { - } 3 \mathrm{~h} \text { à } 800^{\circ} \mathrm{C} \text { - Trempé à l'eau } \overline{20,5} \\
& 3 \mathrm{~h} \text { à } 800^{\circ} \mathrm{C} \quad 24,5 \\
& \mathrm{Te}=29 \begin{array}{l}
\text { Refroidissement au four } \\
3 \mathrm{~h} \text { à } 800^{\circ} \mathrm{C}
\end{array} \\
& \text { Refroidissement au four 22,5 } \\
& +3 \mathrm{~h} \text { à } 800^{\circ} \mathrm{C} \text { - Trempé à l'eau } \\
& 3 \mathrm{~h} \text { à } 800^{\circ} \mathrm{C} \text { - Trempé à l'eau } 31,2 \\
& 3 \mathrm{~h} \text { à } 800^{\circ} \mathrm{C} \text { aur four } \\
& \mathrm{Se}=90 \text { Refroidissement au four } \\
& 3 \text { h à } 800^{\circ} \mathrm{C} \\
& \text { Refroidissement au four } \\
& +3 \text { h à } 800^{\circ} \mathrm{C} \text { - Trempé à l'eau }
\end{aligned}
$$

Il y apparaît que si après refroidissement lent les ségrégations intergranulaires de sélénium et de tellure sont plus importantes qu'après trempe, on retrouve, après un nouveau traitement de trempe, le 
niveau initial des ségrégations obtenu par trempe directe. Nous pouvons donc conclure que la ségrégation intergranulaire du sélénium et du tellure dans le fer pur est un phénomène d'équilibre réversible.

5. Influence d'une ségrégation intergranulaire de carbone. - Comme nous l'avions signalé au début, le carbone joue un rôle bénéfique sur la cohésion des joints de grains. Cet effet a été relié à la présence de ségrégations intergranulaires de carbone mises en évidence par diverses méthodes [16, 17]. Cependant, le mécanisme de consolidation des joints par le carbone n'a jamais été nettement précisé. Deux hypothèses ont été avancées:

- le carbone agit indirectement en prenant aux joints la place des impuretés fragilisantes,

- le carbone a une action directe sur la cohésion des joints.

Pour résoudre ce problème nous avons comparé les ségrégations intergranulaires de sélénium et de tellure dans des fers purs ayant même teneur en sélénium ou tellure mais des teneurs en carbone différentes. Le tableau II rassemble les résultats obtenus.

\section{TABLEAU II}

$\begin{array}{cc}\text { Impuretés } & \begin{array}{c}\text { Ségrégations } \\ (\% \text { de }\end{array} \\ (10-\%) & \begin{array}{c}- \\ \text { monocouche })\end{array} \\ \mathrm{Te}=29 ; \quad \mathrm{C}=10 & 20,5 \\ \mathrm{Te}=31 ; \mathrm{C} \quad 60 & 21,5 \\ \mathrm{Te}=48 ; \mathrm{C}=10 & 38 \\ \mathrm{Te}=48 ; \mathrm{C}=50 & 38,5 \\ \mathrm{Se}=85 ; \mathrm{C}=20 & 29 \\ \mathrm{Se}=90 ; \mathrm{C}=10 & 31 \\ \mathrm{Se}=68 ; \mathrm{C}=10 & 35,5 \\ \mathrm{Se}=70 ; \mathrm{C}=50 & 34,5\end{array}$

Il en ressort que les ségrégations intergranulaires de sélénium et de tellure sont les mêmes en présence ou non de carbone. Comme on l'a vu au paragraphe 2 , ce résultat est valable pour d'autres températures de maintien. On en conclut que le carbone ne chasse pas des joints de grains les impuretés fragilisantes mais ségrège simultanément avec elles et qu'il a sur la cohésion des joints une action intrinsèque dont la nature reste à préciser.

6. Equation de ségrégation et applications. Pour décrire les résultats que nous venons d'évoquer, nous avons cherché à les mettre en équation. Les formules de ségrégation habituellement utilisées [1, 13] s'adaptant mal à nos résultats, nous avons utilisé une 'équation établie dans la référence [18] et qui est de la forme:

$$
\frac{\Gamma}{\Gamma_{\mathrm{M}}}=\frac{A x^{2}}{\left(x-x_{\mathrm{s}}\right)^{2}+A x_{\mathrm{s}}\left(2 x-x_{\mathrm{s}}\right)}
$$

où

$\Gamma$ désigne la ségrégation exprimée en pourcentage de monocouche de fer.

$\Gamma_{\mathrm{M}}$ la ségrégation maximum atteinte pour la saturation des joints de grains.

$x$ la teneur globale en impuretés fragilisantes du fer pur.

$x_{s}$ la limite de solubilité dans le fer pur de cette impureté à la température de ségrégation.

$A$ un terme d'activation thermique de la forme $A=k e^{-Q / R T}$ dépendant de la nature de l'impureté.

Une telle équation permet d'expliquer à la fois les formes en $\mathrm{S}$ des courbes ségrégation/teneur en impuretés, l'existence d'une limite correspondant à la saturation des joints de grains et la loi d'activation thermique des ségrégations.

A titre d'exemple la figure 6 montre l'excellente corrélation obtenue entre les points expérimentaux et la courbe calculée dans le cas des ségrégations intergranulaires de tellure dans des fers traités à $800^{\circ} \mathrm{C}$. Les valeurs utilisées étaient alors :

$$
\begin{aligned}
& \Gamma_{\mathrm{M}}=60 \% \text { de monocouche de fer } \\
& x_{\mathrm{s}}=110 \times 10^{-4} \% \\
& A=0,77
\end{aligned}
$$

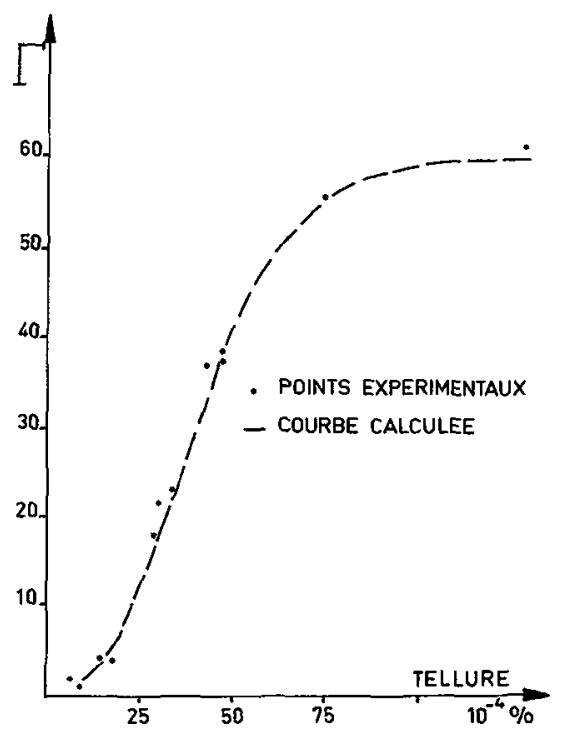

FIG. 6. - Corrélation entre les ségrégations intergranulaires de tellure mesurées expérimentalement et celles déduites de l'équation (1).

Cette équation s'adapte également à certains résultats de la littérature. Ainsi la figure 7 montre quelques résultats de mesures de ségrégations intergranulaires de soufre obtenues par spectroscopie Auger dans des fers traités à $700^{\circ} \mathrm{C}[1,2,3]$. Malgré l'imprécision due au manque de points, on constate que d'une part la courbe ségrégation/teneur globale en soufre a même allure que celle obtenue dans le cas des ségrégations de sélénium et 


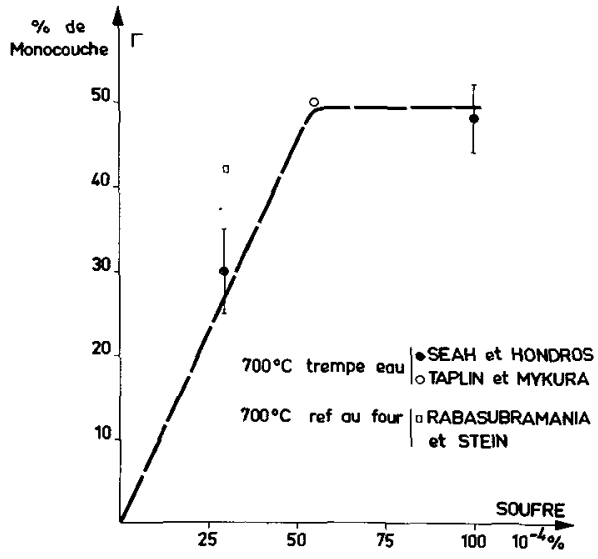

FIG. 7. - Ségrégation intergranulaire du soufre en fonction de sa teneur dans des fers traités à $700^{\circ} \mathrm{C}$ d'après [1], [2] et [3].

de tellure, et que d'autre part les ségrégations intergranulaires de soufre sont plus importantes après refroidissement lent qu'après trempe.

La figure 8 a été obtenue à partir des résultats de Seah et Hondros [1]. Il apparaît que, comme dans le cas des ségrégations de sélénium et de tellure, les ségrégations de soufre suivent une loi d'activation thermique. Il en découle que les ségrégations intergranulaires de soufre peuvent être décrites par la même équation que celles de sélénium et de tellure.

7. Conclusions. - On retiendra essentiellement ici que les ségrégations intergranulaires des éléments soufre, sélénium et tellure dans le fer pur obéissent aux mêmes lois. Il s'agit de ségrégations d'équilibre, réversibles et activées thermiquement.

A température donnée, la forme en $\mathrm{S}$ des courbes ségrégation/teneur globale en impuretés du fer

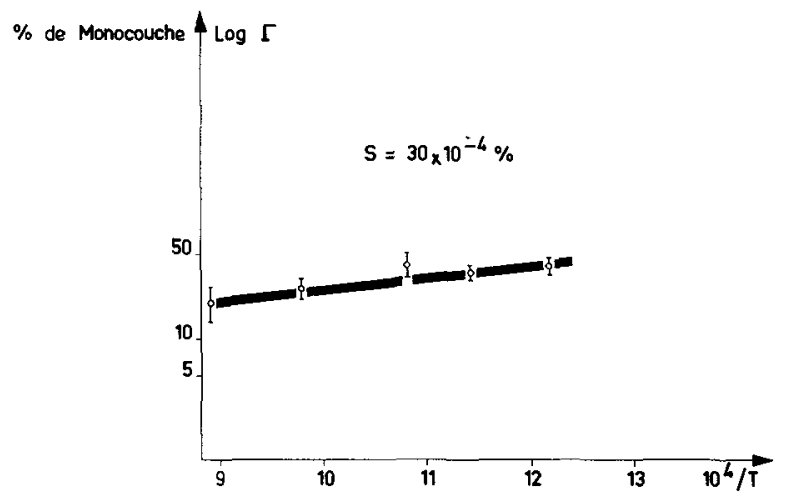

FrG. 8. - Influence de la température de trempe sur la ségrégation intergranulaire du soufre dans le fer d'après [1].

souligne l'analogie qui existe avec l'absorption d'un gaz sur une surface métallique.

La présence de ségrégations intergranulaires de carbone ne modifie pas la quantité d'éléments fragilisants ségrégés, ce qui montre la possibilité de ségrégations intergranulaires simultanées de plusieurs éléments.

Nous proposons enfin une équation générale faisant intervenir la limite de solubilité de l'élément fragilisant dans le fer pur qui s'applique aussi bien à nos résultats qu'à certains résultats de la littérature.

Remerciements. - Nous sommes particulièrement reconnaissants envers $M$. le Professeur Amsel et le Groupe de Physique du Solide de l'Ecole Nationale Supérieure qui nous ont permis d'effectuer les mesures de ségrégations intergranulaires sur leur accélérateur Van de Graff de $2 \mathrm{MeV}$ dans le cadre de la R.C.P. $n^{\circ} 157$ et d'une ATP du C.N.R.S.

\section{Bibliogrsphie}

[1] Seah, M. P. et Hondros, E. D., Proc. R. Soc. Lond. 335 (1973) 191.

[2] Rabasubramanian, P. V. et Stein, D. F., Met. Trans. 4 (1973) 1735

[3] Powell, B. D., Westwood, H. J., Taplin, D. M. R. et MYKura, H., Met. Trans. 4 (1973) 2357.

[4] Guttmann, M., Krahe, P. R., Abel, F., Bruneaux, M. et COHEN, C., Scripta Met. 7 (1973) 93.

[5] Pichard, C., Rieu, J. et Goux, C., Mem. Sci. Rev. Met. 70 (1973) 13.

[6] Pichard, C., Guttmann, M., Rieu, J. et Goux, C., Mem. Sci. Rev. Met., à parâttre.

[7] Guttmann, M., Krahe, P. R., Abel, F., Amsel, G., Bruneaux, M. et Cohen, C., Met. Trans. 5 (1974) 167.

[8] Abel, F., Bull. Soc. Fr. Mineral. Cristallog. 95 (1972) 658.
[9] Farrel, H. H., Isaacs, H. S. et Strongin, M., Surf. Sci. 38 (1973) 31.

[10] MCMAhon, C. J. Jr, Acta Met. 14 (1966) 839.

[11] PICHARD, C., RIEU, J. et GouX, C., Met. Trans., à paraître.

[12] Aust, K. T., Niessen, P., Hanneman, R. E. et WestBROOK, J. H., Acta Met. 16 (1968) 291

[13] MCLEAN, D., Grain Boundaries in Metals (Oxford University Press) 1957, 116.

[14] Faudot, F., Bigot, J. et Talbot-Besnard, S., Mem. Sci. Rev. Met. 69 (1972) 185.

[15] Jolly, P., Mem. Sci. Rev. Met. 66 (1969) 605.

[16] Papazian, J. M. et Beshers, D. N., Met. Trans. 2 (1971) 497.

[17] TURner, P. J. et Papazian, J. M., Met. Sci. J. 7 (1973) 81.

[18] PICHARd, C., Thèse Parıs, à paraitre. 